\title{
Assessment in Work Productivity and the Relationship with Cognitive Symptoms (AtWoRC): primary analysis from a Canadian open-label study of vortioxetine in patients with major depressive disorder (MDD)
}

\author{
Pratap Chokka, ${ }^{1 *}$ Joanna Bougie, ${ }^{2}$ Emmanouil Rampakakis, ${ }^{3}$ and Jean Proul ${ }^{2}$
}

\footnotetext{
${ }^{1}$ Grey Nuns Community Hospital, Edmonton, Alberta, Canada

${ }^{2}$ Lundbeck Canada Inc., Montreal, Quebec, Canada

${ }^{3}$ JSS Medical Research, Montreal, Quebec, Canada
}

Objective. The Assessment in Work Productivity and the Relationship with Cognitive Symptoms (AtWoRC) study aimed to assess the association between cognitive symptoms and work productivity in gainfully employed patients receiving vortioxetine for a major depressive episode (MDE).

Methods. Patients diagnosed with major depressive disorder (MDD) and treated with vortioxetine independently of study enrollment were assessed over 52 weeks at visits that emulated a real-life setting. Patients were classified as those receiving vortioxetine as the first treatment for their current MDE (first treatment) or having shown inadequate response to a previous antidepressant (switch). The primary endpoint was the correlation between changes in patientreported cognitive symptoms (20-item Perceived Deficits Questionnaire [PDQ-D-20]) and changes in work productivity loss (Work Limitations Questionnaire [WLQ]) at week 12. Additional assessments included changes in symptom and disease severity, cognitive performance, functioning, work loss, and safety.

Results. In the week 12 primary analysis, 196 eligible patients at 26 Canadian sites were enrolled, received at least one treatment dose, and attended at least one postbaseline study visit. This analysis demonstrated a significant, strong correlation between PDQ-D-20 and WLQ productivity loss scores $(r=0.634 ; p<0.001)$, and this correlation was significant in both first treatment and switch patients $(p<0.001)$. A weaker correlation between Digit Symbol Substitution Test and WLQ scores was found $(r=-0.244 ; p=0.003)$.

Conclusion. At 12 weeks, improvements in cognitive dysfunction were significantly associated with improvements in workplace productivity in patients with MDD, suggesting a role for vortioxetine in functional recovery in MDD.

Received 15 December 2017; Accepted 21 February 2018; First published online 24 May 2018

Key words: Cognitive dysfunction, functional impairment, major depressive disorder, real life, vortioxetine, work productivity.

\section{Introduction}

Major depressive disorder (MDD) is a highly prevalent, burdensome condition that affects more than 350 million people worldwide. ${ }^{1,2}$ In Canada, the prevalence of MDD was found to be $3.9 \%$ in 2012 , and it is higher in women and younger age groups. $\alpha \mathrm{DD}$ is a multidimensional disease that requires assessment and treatment of

* Address correspondence to: P. Chokka, Grey Nuns Community Hospital, 1100 Youville Drive West, Edmonton, Alberta T6L 5X8, Canada.

(Email: pratapchokka@shaw.ca) various aspects, which include emotional, physical, and cognitive symptoms. ${ }^{4}$ There are more than 1000 combinations of symptoms per the Diagnostic and Statistical Manual of Mental Disorders, 5th ed. (DSM-V), indicating that MDD is a very heterogeneous disease. ${ }^{5}$

Cognitive dysfunction is a core feature of depression, which is ultimately associated with functional impairment and work limitations. ${ }^{6-8}$ In fact, the World Health Organization (WHO) considers depression to be the leading cause of disability worldwide. ${ }^{9}$ Based on previous studies, the estimated prevalence of unemployment and disability in patients with MDD range from $18 \%$ to $34 \%$, 
and the absentee rates range from $23 \%$ to $53 \%$ across Canadian provinces. ${ }^{10}$ In Canada, depression costs more than US $\$ 9$ billion due to lost productivity (i.e., absence from work and attending work while unwell), and depression-related presenteeism (i.e., reduced productivity at work due to MDD) costs US $\$ 6.8$ billion, ${ }^{11}$ demonstrating that MDD is associated with significant economic burden. ${ }^{12}$

The clinical relevance of cognitive dysfunction in MDD and its role in work-related disability is supported by a large body of evidence. ${ }^{6,13-20}$ For the management of adults with MDD, the Canadian Network for Mood and Anxiety Treatments released clinical guidelines in 2016, which state that recovery from depression involves both relief of symptoms and improvement of functioning. ${ }^{4,21}$ However, systematic reviews have shown that improvement in mood symptoms is only modestly correlated with functional outcomes, and few studies of antidepressants have assessed functional outcomes. ${ }^{15,18,22-26}$

Previously, the landmark STAR ${ }^{*} \mathrm{D}$ study, which highlighted the impact of individual depressive symptoms on functional impairment, identified sad mood, concentration, and fatigue as the top three symptoms that have a high relative impact on functional change. ${ }^{27}$ Cognition has been reported to mediate a quarter of the impact of a major depressive episode (MDE) on work loss, ${ }^{18}$ and cognitive symptoms have been associated with greater workplace impairment than depression severity. ${ }^{8}$ Previous studies have reported that greater functional disability and impairment in daily activities were associated with more severe depression and greater perceived cognitive dysfunction, while patients with more severe perceived cognitive dysfunction reported worse work-related productivity outcomes. ${ }^{28}$ Therefore, treatment of cognitive symptoms may hold the key to meaningful functional improvement, yet there is a lack of understanding of the relationship between cognitive dysfunction and functional impairment in MDD. ${ }^{29}$

Vortioxetine is a multimodal antidepressant that is indicated for the treatment of MDD in adults. It differs from selective serotonin reuptake inhibitors and serotonin norepinephrine reuptake inhibitors due to its direct effect on 5-hydroxytryptamine (5-HT) receptors, including $5-\mathrm{HT}_{1 \mathrm{~A}}$ receptor agonism, $5-\mathrm{HT}_{1 \mathrm{~B}}$ receptor partial agonism, and $5-\mathrm{HT}_{3}, 5-\mathrm{HT}_{1 \mathrm{D}}$, and $5-\mathrm{HT}_{7}$ receptor antagonism. ${ }^{30}$

Previous studies have shown that vortioxetine is effective for the treatment of MDD. ${ }^{31,32}$ In addition, studies have shown that vortioxetine is able to improve the cognitive symptoms of MDD. For example, post hoc analyses of a randomized trial in adult patients with MDD who were treated with vortioxetine demonstrated an improvement in the Digital Symbol Substitution Test (DSST), which is an objective cognitive measure of attention/speed of processing, executive functioning, and memory. ${ }^{33}$ The FOCUS study also showed similar improvement in DSST scores in patients treated with vortioxetine. ${ }^{34}$ Moreover, the post hoc analysis from the FOCUS study demonstrated that greater improvement in the measures of cognitive functioning was observed in the working versus total population treated with vortioxetine. ${ }^{35}$ Furthermore, in the CONNECT study, adult patients with recurrent MDD who were treated with vortioxetine had a significant improvement in functional capacity (assessed by the UCSD Performance-Based Skills Assessment [UPSA]) compared with placebo. This study also showed that vortioxetine was superior to placebo in improving the Work Limitations Questionnaire (WLQ) Time Management score, indicating that there may be a relationship between the improvement of cognitive symptoms and the improvement of work productivity with vortioxetine. ${ }^{36}$

Given the established efficacy profile of vortioxetine in improving mood symptoms as well as cognitive symptoms, and the potential relationship between the improvement of cognitive symptoms and work productivity, the Assessment in Work Productivity and the Relationship with Cognitive Symptoms (AtWoRC) study examined the association between cognitive dysfunction and workplace productivity in employed/student patients with MDD treated with vortioxetine. This paper reports results from the 12 -week analysis, including analysis of the primary endpoint.

\section{Methods}

\section{Study design}

The AtWoRC study (NCT02332954) is an interventional, open-label, single-cohort study. It was conducted at 26 sites across Canada by 9 psychiatrists and 17 primary-care physicians. To emulate as closely as possible a real-life setting, structured investigatoradministered interventions and interviews were minimized. The research was approved by the local ethics boards of participating academic sites and the Institutional Review Board Services (Aurora, ON, Canada). Vortioxetine was given at a flexible, open-label dosing of 10-20 mg daily per the Canadian Product Monograph. ${ }^{37}$ Primary endpoint was evaluated at week 12 (analysis after all recruited patients attended the week 12 visit), with follow-ups scheduled every 4 weeks. Patients will then have a follow-up at week 52 (study completion), and a final safety follow-up at week 56 (Figure 1). Data from the final week 52 analysis and from the safety follow-up will be reported elsewhere, as the study is ongoing.

Patients were stratified by treatment history: (1) patients who received vortioxetine as the first treatment for their current MDE (first treatment); and (2) patients who had inadequate response to a previous 


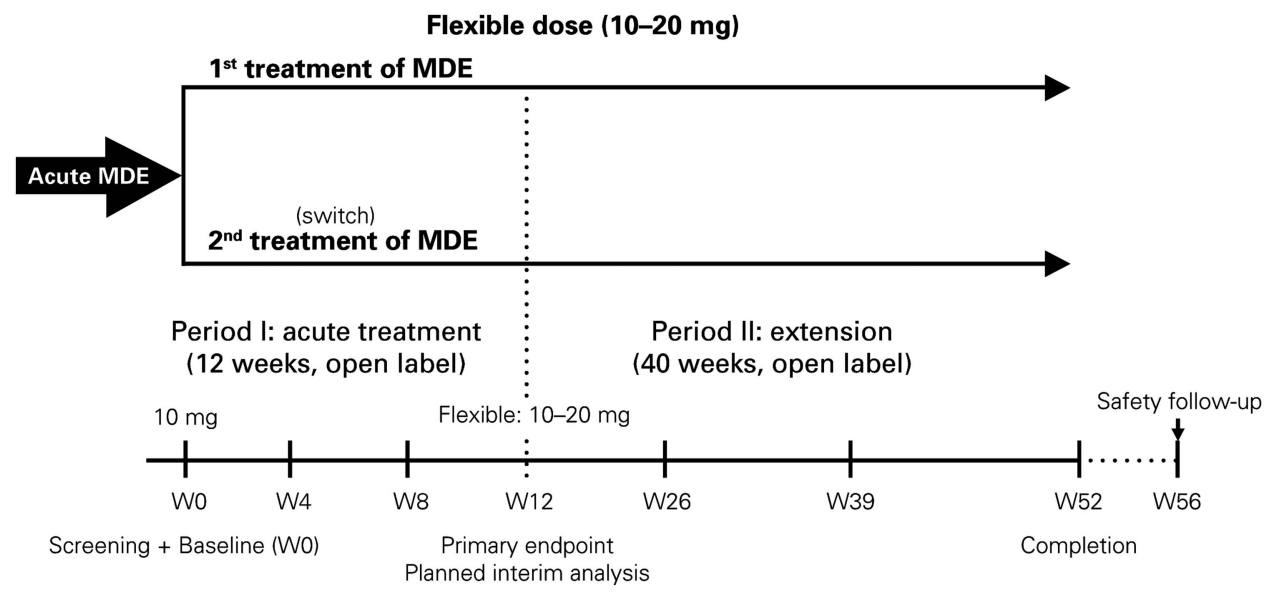

FIGURE 1. Study design of the AtWoRC study. MDE = major depression episode

antidepressant at labeled doses for at least 6 weeks, and it was the opinion of the investigator that treatment with another antidepressant was warranted (switch/ second treatment).

In this study, the Pearson correlation coefficient was the primary statistical parameter used to assess the association between cognitive dysfunction and workplace productivity in patients. Given that this is a single-cohort study, the sample size calculations were driven by the required precision of the correlation coefficient estimate obtained in the study. Precision was assessed with the 95\% confidence interval (CI) of the point estimate (i.e., $95 \%$ CI width of $\pm 20 \%$ of the point estimate). For the outcome measures to be clinically important, a correlation coefficient of $\geq 0.50$ would be required. Therefore, assuming a correlation coefficient of 0.50 and a required $95 \%$ CI of \pm 0.10 (20\% of the point estimate), the total sample size of the study was estimated to be 200 patients.

\section{Assessment tools}

The assessment tools used in the study included patientrated and clinician-rated measures. The patient-rated tools included the Perceived Deficits Questionnaire for Depression (PDQ-D-20), the WLQ productivity loss, the Quick Inventory of Depressive Symptomatology-Self-Report (QIDS-SR), the 12-item WHO Disability Assessment Schedule 2.0 (WHODAS 2.0) questionnaire, the Work Productivity and Activity Impairment (WPAI) questionnaire, the Sheehan Disability Scale (SDS), and the Generalized Anxiety Disorder 7-item (GAD-7) questionnaire. The clinician-rated tools included the Clinical Global Impression-Severity (CGI-S) and CGI-Improvement (CGI-I) scales. The assessment tools also include the DSST (a neuropsychological test). All tools are described in Supplementary Table 1 .

\section{Inclusion and exclusion criteria}

The main inclusion criteria required patients to be between 18 and 65 years of age. A patient's employment status had to be gainfully employed (working $\geq 20$ hours/ week) or enrolled full-time in postsecondary studies or vocational training. The diagnosis of MDD needed to be confirmed according to the DSM-V, and current MDE was confirmed by the investigator, with reported duration of the current MDE of at least 3 months. Patients must have had a baseline score of $\geq 15$ in the QIDS-SR assessment and a baseline score of $\geq 30$ in the PDQ-D-20 assessment.

The main exclusion criteria included a score of $>69$ on the DSST at screening/baseline, to preserve assay sensitivity; current diagnosis or history of manic or hypomanic episode, schizophrenia, or any other psychotic disorder, including major depression with psychotic features; personality disorder, mental retardation, pervasive development disorder, attention-deficit hyperactivity disorder, organic mental disorders, or mental disorders due to a general medical condition (DSM-V criteria); physical, cognitive, or language impairment of such severity as to adversely affect the validity of the data derived from the patient-reported outcomes; current depressive symptoms that were considered by the investigator to have been resistant to two adequate antidepressant treatments of at least 6 weeks duration, each at the maximum recommended dose according to Canadian labeling; and previous exposure to vortioxetine.

\section{Study endpoints}

The primary endpoint of this study was to describe the correlation between change from baseline to week 12 in patient-reported cognitive symptoms (assessed by PDQD-20) and work productivity loss (assessed by WLQ) in gainfully employed patients receiving vortioxetine for an MDE. Baseline depression severity was controlled for in the primary analysis. 
The secondary endpoints were as follows: change in cognitive symptoms and performance (PDQ-D-20 and DSST); change in symptom and disease severity (QIDS-SR, CGI-I, and CGI-S); change in functioning and work productivity (WLQ productivity loss, SDS, WPAI, and WHODAS 2.0); treatment response rate, where response was defined as a change in QIDS-SR of $\geq 50 \%$ from baseline; and remission rate, which was defined as having a QIDS-SR total score of $\leq 5$. Pharmacoeconomic parameters (i.e., work loss) of the whole cohort were described.

Safety and tolerability of vortioxetine were assessed with the incidence of adverse events (AEs), which were coded according to the MedDRA dictionary of terms and were described with the proportion of patients with one or more events within each system organ class and preferred term.

\section{Statistical Analysis}

The study used the following analysis sets: all patients treated set (APTS), which included all patients with a valid baseline assessment who took at least one dose of vortioxetine; and full analysis set (FAS), which included all patients from the APTS who had at least one complete postbaseline study visit.

For the primary endpoint, the correlation between the change from baseline to week 12 in the PDQ-D-20 score and the change from baseline to week 12 in the WLQ productivity loss score was described by the partial correlation coefficient conditional on age, sex, baseline PDQ-D-20, baseline WLQ, disease duration, and disease severity. The analyses were conducted for the 12-week data (first treatment group, switch group, and the total population). To evaluate the impact of missing data at 12 weeks, additional sensitivity analysis was done using the last observation carried forward (LOCF) method.

For the secondary endpoints, the following descriptive statistics were used for the changes from baseline to week 12 in various scores: mean, median, standard deviation $(S D)$, and $95 \%$ CIs of the mean. The rates of response to treatment and remission were described as the proportion of patients achieving these endpoints at week 12 and the corresponding 95\% CIs. Differences between first treatment and switch patients with respect to the baseline and week 12 , and changes from baseline to week 12 in the study outcome parameters were described with the Student $t$ test for independent samples. For functioning and work productivity measures (WLQ, SDS, WPAI, and WHODAS 2.0 scores) and treatment response, all correlations were described with the Pearson correlation and the intraclass correlation coefficient. The secondary correlations (Pearson correlations) were "cross-sectional" at week 12.

\section{Results}

\section{Patient disposition and baseline clinical characteristics}

As of November 2016, 218 patients were enrolled at 26 sites, with 216 eligible patients ( $n=107$ for first treatment, $n=109$ for switch; 2 patients were excluded) who received at least one treatment dose, and formed the APTS. From these patients, 196 patients ( $n=97$ for first treatment, $n=99$ for switch) attended at least one postbaseline study visit and formed the FAS (Figure 2).

At baseline, switch patients were significantly older than those in the first treatment group (38.9 years in first

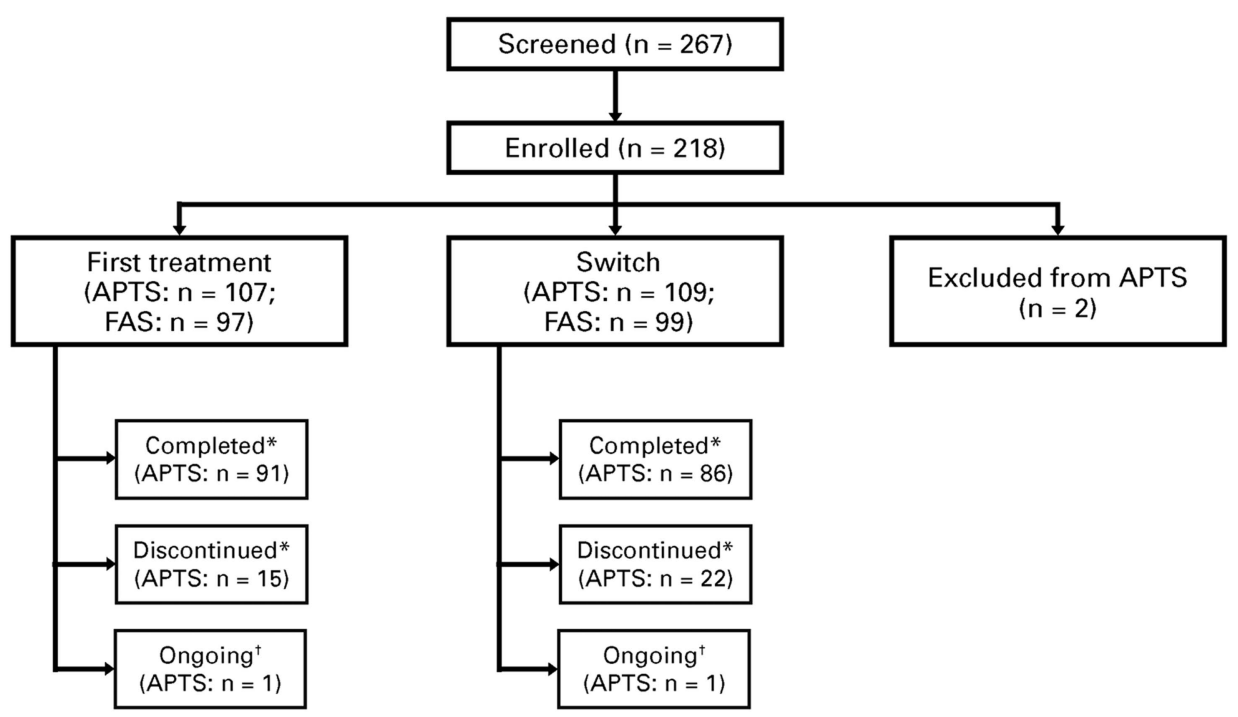

FIGURE 2. Patient disposition. *"Completed" refers to those who attended the Week 12 visit. "Discontinued" refers to treatment discontinuation before Week 12. tOngoing analysis. APTS = all patients treated set; FAS = full analysis set 
TABLE 1. Baseline patient demographics and clinical

characteristics*

\begin{tabular}{lccc} 
& $\begin{array}{c}\text { First treatment } \\
(n=107)\end{array}$ & $\begin{array}{c}\text { Switch } \\
(n=109)\end{array}$ & $\begin{array}{c}\text { Total } \\
\text { (APTS: } n=216)\end{array}$ \\
\hline Mean age, years $(S D) \dagger$ & $38.9(12.7)$ & $42.7(12.0)$ & $40.8(12.4)$ \\
Female gender, \% $(n)$ & $69.2(74)$ & $69.7(76)$ & $69.4(150)$ \\
Caucasian race, \% ( $n)$ & $92.5(99)$ & $95.4(104)$ & $94.0(203)$ \\
Mean duration of disease, & $5.9(6.9)$ & $10.8(10.9)$ & $8.4(9.4)$ \\
$\quad$ years (SD) $\dagger$ & & & \\
Employment type, \% ( $n)$ & & & \\
Employed/independent & $89.7(87)$ & $93.9(93)$ & $91.8(180)$ \\
Full-time vocational & $4.1(4)$ & $1.0(1)$ & $2.6(5)$ \\
Full-time postsecondary student & $6.2(6)$ & $3.0(3)$ & $4.6(9)$ \\
Clinical characteristics: mean scores at baseline & $($ week 0), mean $(S D)$ \\
& First treatment & Switch & Total \\
& $(n=97)$ & $(n=99)$ & $($ FAS: $n=196)$ \\
\hline PDQ-D-20 & $49.7(12.1)$ & $49.5(12.1)$ & $49.6(12.0)$ \\
QIDS-SR & $18.7(2.6)$ & $18.1(2.6)$ & $18.4(2.6)$ \\
GAD-7 $\dagger$ & $15.5(4.7)$ & $14.0(4.8)$ & $14.8(4.8)$ \\
CGI-S & $4.1(0.6)$ & $4.1(0.5)$ & $4.1(0.5)$ \\
WLQ, \% productivity loss & $13.0(4.8)$ & $13.7(4.3)$ & $13.4(4.6)$ \\
WPAl, \% overall impairment & $66.0(23.7)$ & $69.1(22.7)$ & $67.6(23.2)$ \\
SDS & $21.0(4.8)$ & $21.0(5.5)$ & $21.0(5.1)$ \\
WHODAS 2.0 & $21.1(6.8)$ & $21.0(7.9)$ & $21.0(7.4)$ \\
DSST (correct symbols) & $47.5(11.2)$ & $45.0(12.1)$ & $46.2(11.7)$ \\
\hline
\end{tabular}

* For patient demographics, patients in the APTS were assessed. For clinical characteristics, patients in the FAS were assessed.

${ }^{\dagger}$ Significantly different between groups.

APTS = all patients treated set; CGI-S = Clinical Global Impression-Severity; DSST = Digit Symbol Substitution Test; GAD-7 = Generalized Anxiety Disorder 7-item scale; FAS = full analysis set; PDQ-D-20 = Perceived Deficits Questionnaire for Depression; QIDS-SR = Quick Inventory of Depressive SymptomatologySelf-Report; SDS = Sheehan Disability Scale; WHODAS 2.0 $=12$-item World Health Organization Disability Assessment Schedule 2.0; WLQ $=$ Work Limitations Questionnaire; WPAl = Work Productivity and Activity Impairment.

treatment group vs. 42.7 years in switch group; $p=0.024$; Table 1) and had longer disease duration $(p<0.001)$. Over $90 \%$ of patients in both groups were gainfully employed or independent. The mean doses of vortioxetine $(S D)$ at week 12 were $14.6 \mathrm{mg}(5.0), 15.8 \mathrm{mg}$ (5.1), and $15.1 \mathrm{mg}(5.1)$ in the first treatment group, switch group, and total population, respectively.

As shown in Table 1, the mean (SD) PDQ-D-20 scores at baseline were $49.7(12.1)$ and $49.5(12.1)$ in the first treatment and switch groups, respectively. The mean $(S D)$ WLQ productivity loss was $13.0 \%$ (4.8) and $13.7 \%(4.3)$ in the first treatment and switch groups, respectively (the maximum attainable score for the WLQ productivity loss is $25 \%$ ). Overall, patients had severe cognitive dysfunction, severe depression, severe anxiety, and functional impairment. The baseline disease severity was similar in both groups, with the exception of the level of anxiety (GAD-7), which was greater in the first treatment group (15.5 in first treatment vs. 14.0 in switch; $p=0.034$ ).
TABLE 2. Partial correlation between change in PDQ-D-20 and change in WLQ productivity loss from baseline to week $12 *$ (OC)

\begin{tabular}{lrcc} 
Group & $n$ & $r$ & $p$-value \\
\hline OC & & & \\
FAS & 151 & 0.634 & $<0.001$ \\
First treatment & 79 & 0.679 & $<0.001$ \\
Switch & 72 & 0.577 & $<0.001$
\end{tabular}

* Controlled for age, sex, baseline PDQ-D-20, baseline WLQ productivity loss, disease duration, and disease severity (baseline QIDS-SR, baseline CGI-S).

CGI-S = Clinical Global Impression-Severity; FAS = full analysis set; $O C=$ observed cases; PDQ-D-20 = Perceived Deficits Questionnaire for Depression; QIDS-SR = Quick Inventory of Depressive Symptomatology-Self-Report; WLQ = Work Limitations Questionnaire.

\section{Primary endpoint}

In terms of the association between changes from baseline to week 12 in PDQ and WLQ productivity loss, there was a statistically significant, strong correlation between PDQ-D-20 and WLQ scores in the FAS $(r=0.634 ; p<0.001 ;$ Table 2$)$. The results indicate that patients who had improved cognitive function following treatment with vortioxetine also had improved workplace productivity. The correlation between PDQ-D-20 scores and WLQ productivity loss was also significant in both first treatment patients $(r=0.679 ; p<0.001)$ and switch patients $(r=0.577 ; p<0.001)$. To evaluate the impact of missing data at week 12 , additional sensitivity analysis was performed using the LOCF method. The results were similar to those from the observed case analysis (FAS: $r=0.638 ; \quad p<0.001$; first treatment: $r=0.675$; $p<0.001$; switch: $r=0.609$; $p<0.001)$. At week 12 , the largest changes from baseline in the WLQ productivity loss were observed in the time management, mentalinterpersonal demands, and output demands domains (results not shown).

\section{Secondary endpoints}

At week 12, patients treated with vortioxetine showed significant improvement from baseline according to all assessment scores, including measures of cognitive symptoms, disease severity, functional outcomes, work productivity, disability, and objective cognitive performance (Table 3). In addition, the change in DSST was weakly correlated with the change in WLQ productivity loss in the FAS (Table 4). When this analysis is repeated with the removal of the covariates, the correlation becomes statistically significant, although still weak $(r=-0.244 ; p=0.003$; Table 5$)$. The associations between all outcome parameters at week 12 (i.e., the actual test scores) are shown in Supplementary Table 2. 


$\begin{aligned} & \text { TABLE 3. Mean change in different test scores from baseline to } \\
& \text { week } 12 \text { in the FAS (OC)* }\end{aligned}$
\begin{tabular}{lccc} 
Mean (SD) & $\begin{array}{c}\text { First treatment } \\
(n=97)\end{array}$ & $\begin{array}{c}\text { Switch } \\
(n=99)\end{array}$ & $\begin{array}{c}\text { Total (FAS) } \\
(n=196)\end{array}$ \\
\hline PDQ-D-20 & $-23.7(17.3)$ & $-25.5(15.8)$ & $-24.6(16.6)$ \\
QIDS-SR & $-9.8(5.3)$ & $-10.4(5.0)$ & $-10.1(5.2)$ \\
CGI-S & $-1.2(1.1)$ & $-1.1(1.0)$ & $-1.1(1.1)$ \\
WLQ, \% productivity loss & $-6.1(6.3)$ & $-7.1(6.1)$ & $-6.6(6.2)$ \\
WPAI, \% overall impairment & $-31.3(34.6)$ & $-34.3(32.4)$ & $-32.7(33.5)$ \\
SDS & $-10.2(9.0)$ & $-10.8(8.9)$ & $-10.5(8.9)$ \\
WHODAS & $-9.9(9.8)$ & $-10.1(8.9)$ & $-10.0(9.3)$ \\
DSST & $7.8(11.9)$ & $10.1(13.8)$ & $8.9(12.9)$
\end{tabular}

* All changes are $p<0.0001$ (paired $t$ test) compared with baseline. CGI-S = Clinical Global Impression-Severity; DSST = Digit-Symbol Substitution Test; FAS = full analysis set; $\mathrm{OC}=0$ bserved cases; PDQ-D-20 $=$ Perceived Deficits Questionnaire for Depression; QIDS-SR = Quick Inventory of Depressive Symptomatology-Self-Report; SDS $=$ Sheehan Disability Scale; WHODAS 2.0 $=12$-item World Health Organization Disability Assessment Schedule 2.0; WLQ $=$ Work Limitations Questionnaire; WPAI = Work Productivity and Activity Impairment.

TABLE 4. Partial correlation between change in DSST and change in WLQ productivity loss from baseline to week 12 in the FAS (OC)

\begin{tabular}{lcc} 
Group & $r$ & $p$-value \\
\hline Total (FAS) & -0.172 & 0.040 \\
First treatment & -0.255 & 0.032 \\
Switch & -0.084 & 0.513 \\
\hline
\end{tabular}

* Controlled for age, sex, baseline DSST, baseline WLQ productivity loss, disease duration, and disease severity (baseline QIDS-SR, baseline CGI-S).

CGI-S = Clinical Global Impression-Severity; DSST = Digit Symbol Substitution Test; FAS = full analysis set; $O C=$ observed cases; QIDS-SR = Quick Inventory of Depressive Symptomatology-Self-Report; WLQ $=$ Work Limitations Questionnaire.

TABLE 5. Pearson correlation between change in DSST and change in WLQ productivity loss from baseline to week 12 in the FAS

\begin{tabular}{|c|c|c|c|c|c|c|}
\hline & \multicolumn{2}{|c|}{ First treatment } & \multicolumn{2}{|c|}{ Switch } & \multicolumn{2}{|c|}{ FAS } \\
\hline & $r$ & $p$-value* & $r$ & $p$-value* & $r$ & $p$-value* \\
\hline Week 12 & -0.227 & 0.045 & -0.248 & 0.037 & -0.244 & 0.003 \\
\hline
\end{tabular}

${ }^{*} p$-value was calculated using $t$ test for testing of Pearson's correlation coefficient.

DSST $=$ Digit Symbol Substitution Test; FAS $=$ full analysis set; WL $Q=$ Work Limitations Questionnaire.

The treatment response rates at week 12 were $61 \%$ (95\% CI, 51\%-71\%) and 64\% (95\% CI, 54\%-75\%) in the first treatment and switch groups, respectively (Figure 3). There were $28 \%$ (95\% CI, 19\%-37\%) and $39 \%$ (95\% CI, $29 \%-50 \%$ ) of first treatment and switch patients, respectively, who achieved remission at week 12 .

In terms of work loss at 12 weeks, treatment with vortioxetine resulted in fewer patients reporting missing

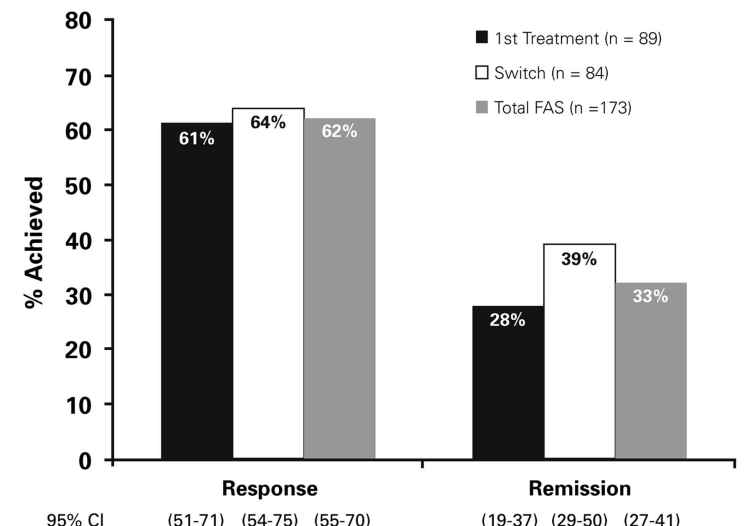

FIGURE 3. Response and remission rates at Week 12 (OC). FAS $=$ Full Analysis Set; $\mathrm{OC}=$ observed cases

\section{TABLE 6. Most common AEs in APTS}

\begin{tabular}{lr} 
Event & \% patients $(n)$ \\
\hline Nausea & $27.8(60)$ \\
Headache & $12.5(27)$ \\
Insomnia & $9.7(21)$ \\
Drug ineffective & $7.4(16)$ \\
Nasopharyngitis & $6.9(15)$ \\
\hline \multicolumn{2}{c}{ AE = adverse event; APTS = all patients treated set. } \\
\hline
\end{tabular}

work due to depression compared with baseline within the previous 3 months $(57 \%$ at baseline vs. $22 \%$ at week 12). In patients who reported missing work at baseline and week 12 visits, fewer missed work days were reported within the previous 3 months at the week 12 visit compared with baseline (12 days at baseline vs. 7 days at week 12; Supplementary Figure 1).

\section{Safety}

The safety profile and tolerability of vortioxetine were consistent with the Canadian product monograph without any new safety signals observed. The most common AEs (frequency $\geq 5 \%$ in APTS, $n=216$ ) were nausea, headache, insomnia, drug ineffectiveness, and nasopharyngitis (Table 6). Withdrawal of consent was the main reason for study discontinuation $(6.5 \%$ of total patient population), and there were 5 patients $(4.7 \%)$ in the first treatment group and 5 patients $(4.6 \%)$ in the switch group who discontinued treatment due to an AE. Other common reasons for treatment discontinuation are outlined in Supplementary Table 3.

\section{Discussion}

At present, there are many studies investigating MDD and response to antidepressant treatment in the clinical setting, but the working MDD patient population has not 
been well studied in a real-life setting. Therefore, it is important to gather practical data on this disorder such that clinical evidence can be translated into the real world and the true benefits of antidepressant treatments in routine clinical care can be demonstrated. Cognitive dysfunction in MDD has been reported to play a role in work productivity impairment. The 12 -week analysis of the AtWoRC study demonstrated that improvements in cognitive function were strongly correlated with improvements in workplace productivity in gainfully employed patients with MDD receiving vortioxetine in a real-life setting.

As shown in Table 2, changes in PDQ-D-20 scores were found to be significantly correlated with WLQ productivity loss scores. These results suggest that treatment of perceived cognitive symptoms with vortioxetine may increase workplace productivity. Given that returning to optimal levels of gainful employment is an essential element of patient re-integration, and given that the patients in this study improved globally on all investigated measures, including those assessing cognitive symptoms, these results suggest that vortioxetine is an effective option.

After 12 weeks of treatment with vortioxetine, patients showed significant improvements in perceived cognitive symptoms and cognitive performance (as shown by PDQ-D-20 and DSST, respectively), symptoms and disease severity (as shown by QIDS, CGI-S), work productivity (as shown by WLQ productivity loss and WPAI), and functioning (as shown by SDS and WHODAS 2.0; Table 3). The improvement in objective cognitive performance as assessed by the DSST and perceived cognitive dysfunction as assessed by the PDQ-D-20 were consistent with previous studies with vortioxetine. ${ }^{31,32,34,36}$ Moreover, results from the AtWoRC study demonstrated the efficacy of vortioxetine in working patients, which supported previous findings from the post hoc analysis of the FOCUS study that showed a significant improvement in the DSST scores with vortioxetine at 10 and $20 \mathrm{mg}$ in the working patients $(p<0.001)$. The analysis also showed that vortioxetine had a greater effect in working patients when compared with the total study population; a similar pattern was observed for the PDQ and Montgomery-Åsberg Depression Rating Scale scores. ${ }^{35}$ In addition, in the CONNECT study, vortioxetine was superior to placebo in the improvement of WLQ-Time Management score, indicating that there may be a correlation between improvement of cognitive symptoms and improvement in work productivity. Indeed, this relationship was examined and demonstrated in the AtWoRC study. Overall, the large-scale, placebo-controlled studies showed that patients treated with vortioxetine have consistent improvement in cognitive function. The present study reinforces these results in a real-life setting while highlighting the association between cognitive symptoms and work productivity.

Comparing with previous studies with vortioxetine, the response and remission rates were high, which were consistent with previous studies with vortioxetine. ${ }^{30,38,39}$

In the past, very few studies have explored the association between the change in perceived cognitive symptoms and change in workplace productivity. A previous report from the Combined Medications to Enhance Depression Outcomes (CO-MED) trial demonstrated this association by assessing perceived cognitive symptoms with QIDS-Clinician Rated and QIDS-SR, and work productivity with WPAI and the Massachusetts General Hospital Cognitive and Physical Functioning Questionnaire. ${ }^{40}$ The CO-MED trial also showed that patients with improved self-reported work productivity had 3 to 5 times greater remission rates at 3 months posttreatment. The AtWoRC study is one of the few studies that explored the association in a Canadian real-life setting, and it further validates and consolidates previous findings, including those from the CO-MED trial.

Despite the correlation between changes in subjective cognitive symptoms (PDQ-D-20) and work productivity (WLQ productivity loss) found in this study, only a weak correlation between changes in objective cognitive performance (DSST) and work functioning (WLQ productivity loss) was observed for the total patient population in the FAS. If the covariates were removed, there was a slightly stronger and statistically significant correlation between changes in DSST and WLQ productivity loss scores (Table 5), though the correlation was still weak. From these results, it is speculated that there are differences between subjective- and objective-rated cognitive assessment tools. In the study by Srisurapanont et al., discrepancy was found between subjective and objective measures of cognition in patients with MDD, and the authors concluded that age and depression severity might predict the discrepancy between the two sets of measures. ${ }^{41}$ When Lam et al. examined the effect of desvenlafaxine on neurocognitive and work functioning in working patients with MDD, they showed similar findings, in that there were no significant correlations between changes in objective cognitive function and changes in measures of work functioning. ${ }^{42}$ In another study, subjective and objective measures of cognition were also reported not to be associated with each other. ${ }^{43}$ Subjective cognitive impairment, but not objective cognitive impairment, was found to be significantly associated with psychosocial impairment; this difference might be attributed to differences in depression severity.

While the pharmacoeconomic parameters were descriptive in nature, improvements were indeed observed in the number of patients who missed work days and in the number of work days missed from 
baseline to week 12. This finding supports the primary endpoint result in that treatment with vortioxetine may also lead to reduced presenteeism in the workplace. Accordingly, this result highlights the importance of treating MDD in order to reduce its economic burden in addition to its clinical burden.

Overall, the safety profile of vortioxetine was consistent with previous studies and the product monograph. Common AEs included nausea, headache, and diarrhea, demonstrating that vortioxetine was well tolerated in the patient population. For first treatment and switch groups, the rate of discontinuation due to adverse drug reaction at week 12 was low $(4.7 \%$ and $4.6 \%$, respectively) compared with other open-label studies $(11.3 \%$ with duloxetine as first treatment ${ }^{44} ; 6.3 \%$ when switched to duloxetine $\mathrm{e}^{45}$ ). The most common reason for discontinuation in all groups was withdrawal of consent. There were 5 patients in the first treatment group and 5 patients in the switch group who discontinued treatment due to loss of follow-up. It is speculated that since patients in the AtWoRC study were mostly gainfully employed, study assessments might add burden to their well-being, which could lead to discontinuation of treatment.

There were a few limitations to this study, which included its open-label nature and potential placebo effect. However, it should be noted that the primary objective of the study was to evaluate the correlation between improvement in perceived cognitive symptoms and work productivity, and treatment effectiveness was assessed secondarily. A control group or a comparative treatment was also lacking. Hence, the improvements in the test scores observed in this study could not be directly attributed to a study or drug effect.

\section{Conclusions}

In this 12-week analysis of the AtWoRC study, patients with depression who were treated with vortioxetine had improvements in cognitive function and improved workplace productivity. After 12 weeks of treatment with vortioxetine, both first treatment and switch patients showed significant improvements in cognitive symptoms and performance, disease severity, work productivity, and functioning; the results are consistent with previous studies with vortioxetine. The real-life data from the AtWoRC study confirmed that vortioxetine is an efficacious, safe, and well-tolerated treatment option for patients with MDD. Future results from the 52-week final analysis follow-up are expected later in 2018.

\section{Acknowledgments}

Under the direction of Dr. Chokka, Dr. Bougie, Dr. Rampakakis, and Mr. Proulx, medical writing assistance was provided by Jane Cheung, PhD, of SAGE Medica Inc.; this assistance was supported by Lundbeck Canada. All authors were involved at all stages of manuscript development, approved the final version of the manuscript to be published, and agreed to act as guarantors of the work. We would also like to thank the following principal investigators at the participating sites: Dr. Sunny Johnson, Dr. Paul Latimer, Dr. Brian Ramjattan, Dr. Mark Johnston, Dr. Michael O'Mahony, Dr. Sean Peterson, Dr. William O’Mahony, Dr. Murray Awde, Dr. Michael Csanadi, Dr. Ranjith Chandrasena, Dr. Preston Zuliani, Dr. Peter Turner, Dr. Richard Tytus, Dr. Jeannette Janzen, Dr. Guy Chouinard, Dr. Giuseppe Mazza, Dr. Denis Beaulieu, Dr. Valérie Tourjman, Dr. François Blouin, Dr. MarieChantal Ménard, Dr. Benicio Frey, Dr. Hani Iskandar, Dr. Arun Nayar, Dr. Jasmin Belle-Isle, and Dr. Ethel Bellavance.

\section{Funding}

This study was supported by Lundbeck Canada.

\section{Disclosures}

Pratap Chokka has received honoraria from serving on advisory boards with Allergan, Lundbeck, Janssen, Pfizer, Sunovion, and Shire. He also had speaking engagements with Allergan, Lundbeck, Janssen, Pfizer, Sunovion, Shire, and Purdue. He received research grants from Lundbeck and Janssen. Joanna Bougie and Jean Proulx are employees of Lundbeck Canada. Emmanouil Rampakakis is an employee of JSS Medical Research, the contract research organization mandated to manage the study. The authors declare no conflicts of interest in this work.

\section{Supplementary material}

To view supplementary material for this article, please visit https://doi.org/10.1017/S1092852918000913

\section{REFERENCES:}

1. Rosenblat JD, Kakar R, McIntyre RS. The cognitive effects of antidepressants in major depressive disorder: A systematic review and meta-analysis of randomized clinical trials. International Journal of Neuropsychopharmacology. 2015; 19(2).

2. Kessler RC, Akiskal HS, Ames M, et al. Prevalence and effects of mood disorders on work performance in a nationally representative sample of U.S. Workers. Am J Psychiatry. 2006; 163(9): 1561-1568.

3. Patten SB, Williams JV, Lavorato DH, et al. Descriptive epidemiology of major depressive disorder in Canada in 2012. Can J Psychiatry. $2015 ; 60(1): 23-30$.

4. Lam RW, McIntosh D, Wang J, et al. Canadian Network for Mood and Anxiety Treatments (CANMAT) 2016 clinical guidelines for the management of adults with major depressive disorder: Section 1. 
Disease burden and principles of care. Can J Psychiatry. 2016; 61(9): 510-523.

5. Ostergaard SD, Jensen SO, Bech P. The heterogeneity of the depressive syndrome: when numbers get serious. Acta Psychiatr Scand. 2011; 124(6): 495-496.

6. McIntyre RS, Cha DS, Soczynska JK, et al. Cognitive deficits and functional outcomes in major depressive disorder: determinants, substrates, and treatment interventions. Depress Anxiety. 2013; 30(6): 515-527.

7. Evans VC, Iverson GL, Yatham LN, et al. The relationship between neurocognitive and psychosocial functioning in major depressive disorder: a systematic review. J Clin Psychiatry. 2014; 75(12): 1359-1370.

8. McIntyre RS, Soczynska JZ, Woldeyohannes HO, et al. The impact of cognitive impairment on perceived workforce performance: results from the International Mood Disorders Collaborative Project. Compr Psychiatry. 2015; 56: 279-282.

9. World Health Organization. Depression fact sheet. 2017. http:// www.who.int/mediacentre/factsheets/fs369/en. Accessed October 5, 2017.

10. Rizvi SJ, Cyriac A, Grima E, et al. Depression and employment status in primary and tertiary care settings. Can J Psychiatry. 2015; 60(1): 14-22.

11. Evans-Lacko S, Knapp M. Global patterns of workplace productivity for people with depression: absenteeism and presenteeism costs across eight diverse countries. Soc Psychiatry Psychiatr Epidemiol. 2016; 51(11): 1525-1537.

12. Greenberg PE, Fournier AA, Sisitsky T, et al. The economic burden of adults with major depressive disorder in the United States (2005 and 2010). J Clin Psychiatry. 2015; 76(2): 155-162.

13. Veiel HO. A preliminary profile of neuropsychological deficits associated with major depression. J Clin Exp Neuropsychol. 1997; 19(4): 587-603.

14. Goeldner C, Ballard TM, Knoflach F, et al. Cognitive impairment in major depression and the mglu2 receptor as a therapeutic target. Neuropharmacology. 2013; 64: 337-346.

15. Lee RS, Hermens DF, Porter MA, et al. A meta-analysis of cognitive deficits in first-episode major depressive disorder. J Affect Disord. 2012; 140(2): 113-124.

16. Hasselbalch BJ, Knorr U, Kessing LV. Cognitive impairment in the remitted state of unipolar depressive disorder: a systematic review. J Affect Disord. 2011; 134(1-3): 20-31.

17. Weisenbach SL, Boore LA, Kales HC. Depression and cognitive impairment in older adults. Curr Psychiatry Rep. 2012; 14(4): 280-288.

18. Buist-Bouwman MA, Ormel J, de Graaf R, et al. Mediators of the association between depression and role functioning. Acta Psychiatr Scand. 2008; 118(6): 451-458.

19. Jaeger J, Berns S, Uzelac S, et al. Neurocognitive deficits and disability in major depressive disorder. Psychiatry Res. 2006; 145(1): 39-48.

20. Southerland G, Stonebridge C. Healthy Brains at Work: Estimating the Impact of Workplace Mental Health Benefits and Programs. Ottawa: The Conference Board of Canada; 2016.

21. Kennedy SH, Lam RW, McIntyre RS, et al. Canadian Network for Mood and Anxiety Treatments (CANMAT) 2016 clinical guidelines for the management of adults with major depressive disorder: Section 3. Pharmacological treatments. Can J Psychiatry. 2016; 61(9): 540-560.

22. McIntyre RS, Lee Y, Mansur RB. Treating to target in major depressive disorder: response to remission to functional recovery. CNS Spectr. 2015; 20(Suppl 1): 20-30; quiz 31.

23. Campbell S, Macqueen G. The role of the hippocampus in the pathophysiology of major depression. J Psychiatry Neurosci. 2004; 29(6): 417-426.
24. Murrough JW, Iacoviello B, Neumeister A, et al. Cognitive dysfunction in depression: neurocircuitry and new therapeutic strategies. Neurobiol Learn Mem. 2011; 96(4): $553-563$.

25. Harmer CJ, Clark L, Grayson L, et al. Sustained attention deficit in bipolar disorder is not a working memory impairment in disguise. Neuropsychologia. 2002; 40(9): 1586-1590.

26. Iosifescu DV. The relation between mood, cognition and psychosocial functioning in psychiatric disorders. Eur Neuropsychopharmacol. 2012; 22(Suppl 3): S499-S504.

27. Fried EI, Nesse RM. The impact of individual depressive symptoms on impairment of psychosocial functioning. PLoS One. 2014; 9(2): e90311.

28. Kim JM, Chalem Y, di Nicola S, et al. A cross-sectional study of functional disabilities and perceived cognitive dysfunction in patients with major depressive disorder in South Korea: the PERFORM-K study. Psychiatry Res. 2016; 239: 353-361.

29. Francomano A, Bonanno B, Fuca L, et al. The role of antidepressant treatments on cognitive deficits. A review of recent literature. Clinical Neuropsychiatry. 2011; 8(6): 354-366.

30. Garnock-Jones KP. Vortioxetine: a review of its use in major depressive disorder. CNS Drugs. 2014; 28(9): 855-874.

31. Thase ME, Mahableshwarkar AR, Dragheim M, et al. A meta-analysis of randomized, placebo-controlled trials of vortioxetine for the treatment of major depressive disorder in adults. Eur Neuropsychopharmacol. 2016; 26(6): 979-993.

32. Katona C, Hansen T, Olsen CK. A randomized, double-blind, placebo-controlled, duloxetine-referenced, fixed-dose study comparing the efficacy and safety of lu aa21004 in elderly patients with major depressive disorder. Int Clin Psychopharmacol. 2012; 27(4): 215-223.

33. Harrison JE, Lophaven S, Olsen CK. Which cognitive domains are improved by treatment with vortioxetine? International Journal of Neuropsychopharmacology. 2016.

34. McIntyre RS, Lophaven S, Olsen CK. A randomized, double-blind, placebo-controlled study of vortioxetine on cognitive function in depressed adults. International Journal of Neuropsychopharmacology. 2014; 17(10): 1557-1567.

35. McIntyre RS, Florea I, Tonnoir B, et al. Efficacy of vortioxetine on cognitive functioning in working patients with major depressive disorder. J Clin Psychiatry. 2017; 78(1): 115-121.

36. Mahableshwarkar AR, Zajecka J, Jacobson W, et al. A randomized, placebo-controlled, active-reference, double-blind, flexible-dose study of the efficacy of vortioxetine on cognitive function in major depressive disorder. Neuropsychopharmacology. 2015; 40(8): 2025-2037.

37. Trintellix $(\mathrm{tm})$ (vortioxetine) product monograph. Montreal, QC, Canada: Lundbeck Canada Inc.; 2014.

38. Boulenger JP, Loft H, Olsen CK. Efficacy and safety of vortioxetine (lu aa21004), 15 and $20 \mathrm{mg} /$ day: a randomized, double-blind, placebo-controlled, duloxetine-referenced study in the acute treatment of adult patients with major depressive disorder. Int Clin Psychopharmacol. 2014; 29(3): 138-149.

39. Henigsberg N, Mahableshwarkar AR, Jacobsen $\mathrm{P}$, et al. A randomized, double-blind, placebo-controlled 8-week trial of the efficacy and tolerability of multiple doses of lu aa21004 in adults with major depressive disorder. J Clin Psychiatry. 2012; 73(7): 953-959.

40. Jha MK, Minhajuddin A, Greer TL, et al. Early improvement in work productivity predicts future clinical course in depressed outpatients: findings from the CO-MED trial. Am J Psychiatry. 2016; 173(12): 1196-1204.

41. Srisurapanont M, Suttajit S, Eurviriyanukul K, et al. Discrepancy between objective and subjective cognition in adults with major depressive disorder. Scientific Reports. 2017; 7(1): 3901. 
42. Lam RW, Iverson GL, Evans VC, et al. The effects of desvenlafaxine on neurocognitive and work functioning in employed outpatients with major depressive disorder. J Affect Disord. 2016; 203: 55-61.

43. Cha DS, Carmona NE, Subramaniapillai M, et al. Cognitive impairment as measured by the thinc-integrated tool (thinc-it): Association with psychosocial function in major depressive disorder. J Affect Disord. 2017; 222: 14-20.

44. Hudson JI, Perahia DG, Gilaberte I, et al. Duloxetine in the treatment of major depressive disorder: an open-label study. BMC Psychiatry. 2007; 7: 43.

45. Wohlreich MM, Martinez JM, Mallinckrodt CH, et al. An open-label study of duloxetine for the treatment of major depressive disorder: comparison of switching versus initiating treatment approaches. Journal of Clinical Psychopharmacology. 2005; 25(6): 552-560.

46. Lawrence C, Roy A, Harikrishnan V, et al. Association between severity of depression and self-perceived cognitive difficulties among full-time employees. Prim Care Companion CNS Disord. $2013 ; 15(3)$.

47. Lam RW, Saragoussi D, Danchenko N, et al. Psychometric validation of Perceived Deficits Questionnaire-Depression (PDQ-D) in patients with major depressive disorder (MDD). Value in Health. 2013; 16(7): A330.

48. Lerner D, Adler DA, Chang H, et al. The clinical and occupational correlates of work productivity loss among employed patients with depression. J Occup Environ Med. 2004; 46(6 Suppl): S46-S55.

49. Tang K, Beaton DE, Boonen A, et al. Measures of work disability and productivity: Rheumatoid Arthritis Specific Work Productivity Survey (WPS-RA), Workplace Activity Limitations Scale (WALS), Work Instability Scale for Rheumatoid Arthritis (RA-WIS), Work Limitations Questionnaire (WLQ), and Work Productivity and Activity Impairment Questionnaire (WPAI). Arthritis Care Res (Hoboken). 2011; 63(Suppl 11): S337-S349.
50. Rush AJ, Trivedi MH, Ibrahim HM, et al. The 16-item Quick Inventory Of Depressive Symptomatology (QIDS), Clinician Rating (QIDS-C), and Self-Report (QIDS-SR): a psychometric evaluation in patients with chronic major depression. Biol Psychiatry. 2003; 54(5): 573-583.

51. Ustun TB, Chatterji S, Kostanjsek N, et al. Developing the World Health Organization Disability Assessment Schedule 2.0. Bulletin of the World Health Organization. 2010; 88(11): 815-823.

52. Reilly MC, Zbrozek AS, Dukes EM. The validity and reproducibility of a work productivity and activity impairment instrument. Pharmacoeconomics. 1993; 4(5): 353-365.

53. Trivedi MH, Morris DW, Wisniewski SR, et al. Increase in work productivity of depressed individuals with improvement in depressive symptom severity. Am J Psychiatry. 2013; 170(6): 633-641.

54. Arbuckle R, Frye MA, Brecher M, et al. The psychometric validation of the Sheehan Disability Scale (SDS) in patients with bipolar disorder. Psychiatry Res. 2009; 165(1-2): 163-174.

55. Lowe B, Decker O, Muller S, et al. Validation and standardization of the Generalized Anxiety Disorder Screener (GAD-7) in the general population. Med Care. 2008; 46(3): 266-274.

56. Guy W. ECDEU Assessment Manual for Psychopharmacology. Rockville, MD: US Department of Health, Education, and Welfare; 2014.

57. Swan GE, Carmelli D, LaRue A. Performance on the Digit Symbol Substitution Test and 5-year mortality in the western collaborative group study. Am J Epidemiol. 1995; 141(1): 32-40.

58. Rosano C, Newman AB, Katz R, et al. Association between lower Digit Symbol Substitution Test score and slower gait and greater risk of mortality and of developing incident disability in well-functioning older adults. J Am Geriatr Soc. 2008; 56(9): 1618-1625. 